\title{
Expected Duration of Adverse Pregnancy Outcomes after Zika Epidemic
}

\section{Rosalind M. Eggo, Adam J. Kucharski}

Evidence is increasing that Zika virus-related adverse outcomes can occur throughout pregnancy. Mathematical modeling analysis using reported outcome data suggests that surveillance for these outcomes should begin as soon as an outbreak is detected and should continue for 40 weeks after the outbreak ends.

$\mathrm{Q}$ uantifying the risk for adverse pregnancy outcomes (APOs) after Zika virus infection is of critical public health importance. Recent studies have suggested that risk for microcephaly is concentrated in pregnancies in which infection occurs during the first trimester $(1,2)$. However, microcephaly is at the severe end of the APO spectrum and might have a different risk profile from other outcomes: brain abnormality and malformation, eye anomalies, neural tube defects, arthrogryposis, congenital deafness, and others (3). In particular, estimates of APOs after symptomatic confirmed Zika virus infection suggest risk for fetal injury throughout pregnancy $(3,4)$. Thus, a better understanding of the likely duration and risk for APOs after Zika virus outbreaks is urgently needed (2). We used surveillance and clinical data to estimate the timing and number of expected APO events after observed Zika outbreaks in 9 regions of Brazil during April 2015-July 2017.

\section{The Study}

To quantify APO risk, we used data from a study that recruited 345 pregnant women with rash in the previous 5 days, of whom 134 tested positive for Zika virus infection (4). Excluding 9 losses to follow-up, we followed a cohort of 125 women during pregnancy; surviving infants were examined for APOs. A total of 58 APOs occurred in this group; microcephaly was infrequent (4 [6.9\%] of 58) but severe (4).

We fitted a logistic model to individual-level data for the 125 followed-up women to estimate the proportion of APOs after symptomatic Zika virus infection at each week of gestation (Figure 1, panel A). Although the fitted linear model suggested a decline in risk over time, the model did not perform significantly better than a model with constant

Affiliation: London School of Hygiene \& Tropical Medicine, London, UK

DOI: https://doi.org/10.3201/eid2401.170482 risk for APO at any gestational age (online Technical Appendix, https://wwwnc.cdc.gov/EID/article/24/1/17-0482Techapp1.pdf). For comparison, we also considered a theoretical risk profile in which APO risk occurs only during the first trimester (Figure 1, panel A).

We used these risk profiles to estimate the period through which an elevated rate of APOs would be expected after the 2015-2016 Zika epidemic in 9 regions of Brazil (Figure 1, panels B-J). We superimposed the timing of confirmed microcephaly cases in each region to assess the relationship between observed microcephaly and expected duration of elevated APO risk but did not fit explicitly to microcephaly incidence data. If risk were assumed to occur only during the first trimester, the period of APOs would be shorter than the duration of observed microcephaly events. In contrast, the predicted durations of APOs based on risk throughout pregnancy were more consistent with the observed distribution of microcephaly in these regions.

To examine the potential duration and risk for Zikaassociated APOs more generally, we also predicted the pattern of APOs under 3 hypothetical epidemic scenarios: single outbreak, multipeaked epidemic, and endemic transmission (Figure 2). For each epidemic scenario, the model suggested that the duration of elevated risk was much longer than the duration of cases if APOs could occur from infection in any gestational week. This observation means that in areas where seasonal outbreaks of Zika occur, the risk for APOs might not return to baseline levels between epidemics, and Zika-specific interventions based on timing of pregnancy might be less effective (7).

Our findings are subject to several limitations. First, we based the estimation of APO risk by gestation period on a cohort study of symptomatic infection with rash, which does not occur with all Zika virus infections (8). However, recent evidence suggests the risk for APOs is similar for symptomatic and asymptomatic infection (9). We included pregnancy loss during the first trimester (miscarriage) as an APO, but excluding these 5 cases did not alter the findings (online Technical Appendix, Sensitivity Analysis on Inclusion of Miscarriages section). Moreover, evidence suggests that APOs might not be detectable at birth but appear later, which would underestimate the frequency of APOs (10).

Second, the data were from patients recruited in Rio de Janeiro, whereas we considered potential risk across all regions of Brazil. Although the cohort was large and APO data detailed, numbers of exposed women in each gestational 
week were low, leading to large CIs on the risk profile (Figure 1, panel A). We therefore used a linear model to estimate the risk at each gestational week because data were insufficient to fit a more complex risk function. The range of data (6-39 weeks' gestation) also constrained our estimates.

Third, publicly available epidemiologic reports from Brazil recorded microcephaly cases, rather than all forms of APO. We qualitatively compared these microcephaly reports with our estimates for the duration of risk for APOs, but the risk for microcephaly by gestational week might differ from the overall risk for APOs. Different regions are likely to have differing baseline levels of APOs in the absence of Zika virus infection; we therefore focused our analysis on the risk for APOs associated with Zika virus
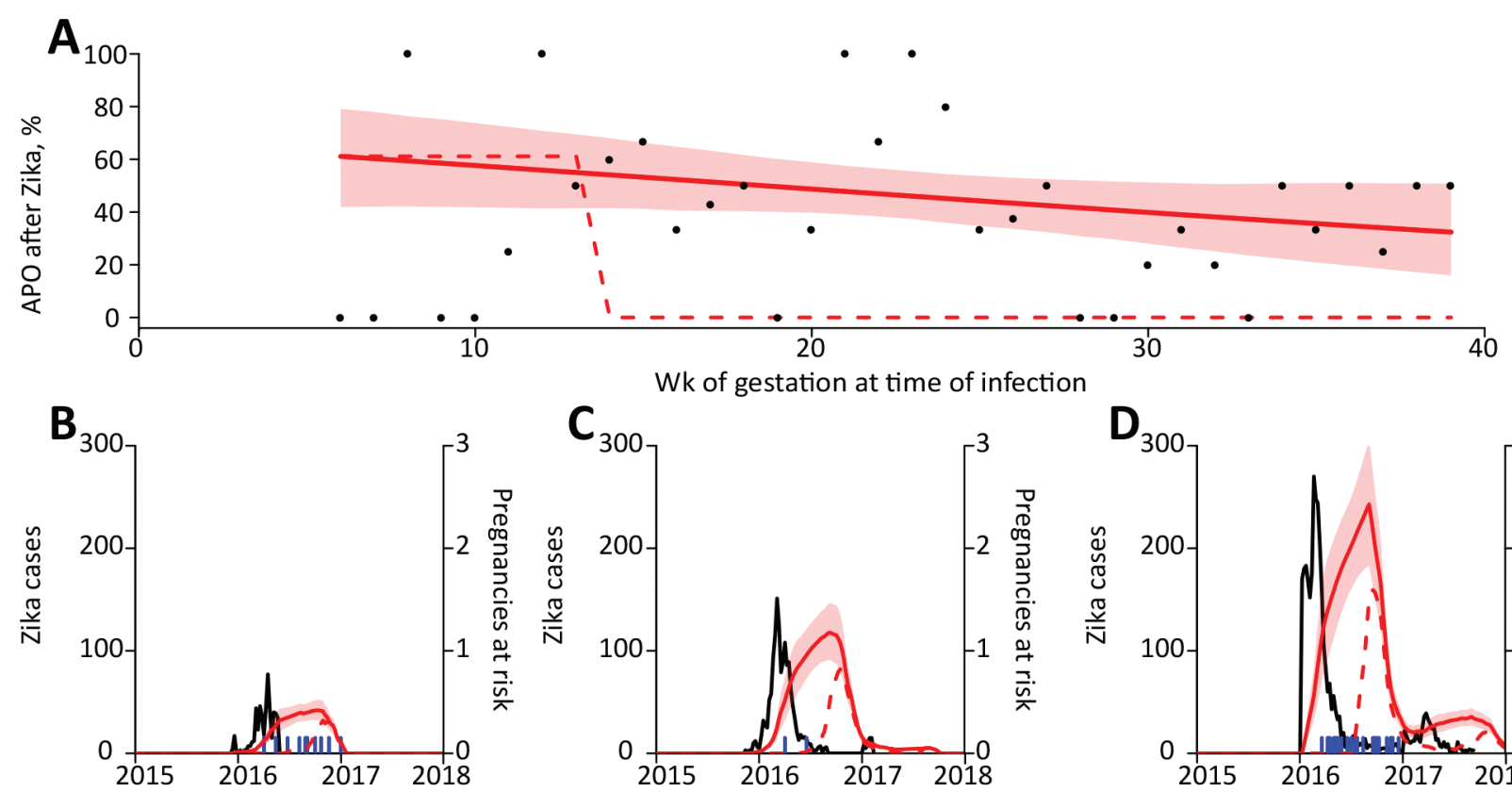

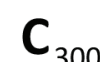

Wk of gestation at time of infection
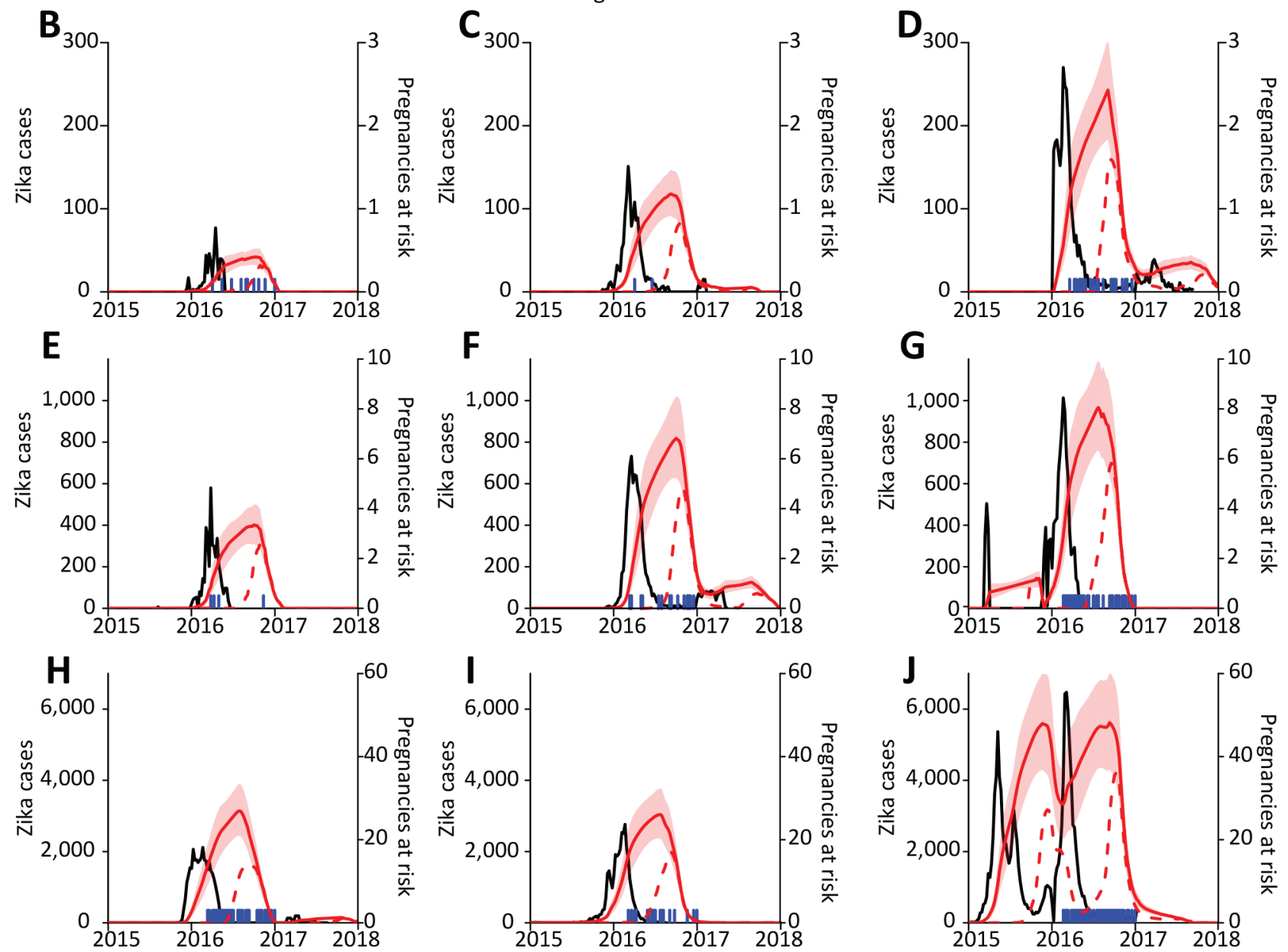

Figure 1. Relationship between Zika virus infection and expected related APOs per 1,000 pregnancies in Brazil during April $2015-J u l y ~ 2017$. A) Percentage of APOs (fetal loss at any gestational age, stillbirth, neonatal abnormality) given symptomatic PCR-confirmed Zika virus infection. Points show weekly proportion with APO (4); red line indicates fit to data with a generalized linear model, and shading indicates $95 \% \mathrm{Cls}$; dashed line indicates fixed risk in first trimester only (5). B-J) Blue lines indicate suspected Zika cases in different regions; red lines indicate expected number of births with Zika-associated APO in subsequent weeks based on the 2 risk distributions in panel A. Shaded regions indicate $95 \% \mathrm{Cls}$. Model assumes $17 \%$ of Zika virus infections are reported $(5,6)$. APO, adverse pregnancy outcome. 


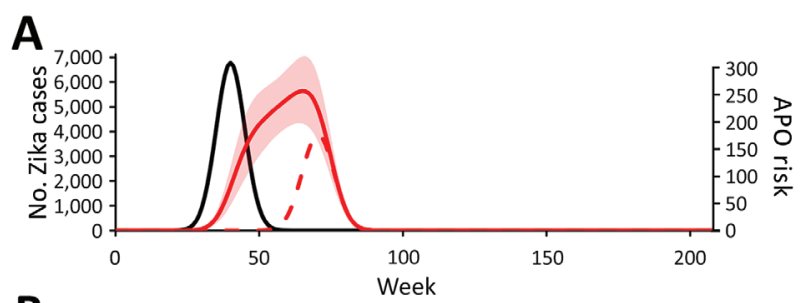

B
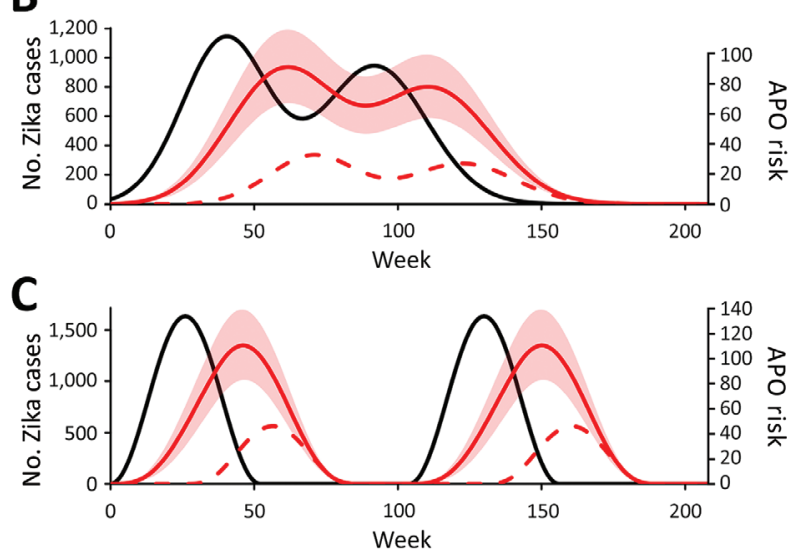

Figure 2. Expected temporal distribution of Zika virus-related adverse pregnancy outcomes under different hypothetical outbreak scenarios, Brazil, April 2015-July 2017. Black lines indicate Zika cases; red lines indicate risk (APOs/1,000 births) for Zika-associated APO in subsequent weeks based on the 2 risk distributions in panel $A$. Dashed lines indicate timing of outbreaks: A) short, single-peaked outbreak; B) double-peaked outbreak; C) biennial epidemics (i.e., a seasonal endemic state). A population size of 1 million, reporting of $17 \%$ of Zika infections, and a $50 \%$ attack rate during a 4-year period were assumed. APO, adverse pregnancy outcome.

infection. Why some areas of Latin America have reported more cases of microcephaly than others remains unclear (11). There may be unmeasured cofactors that alter the risk for APO on Zika virus infection (12). Another factor could be differences in the proportion of Zika cases reported, which could lead to variation in incidence of APOs. We assumed $17 \%$ of Zika infections were reported $(6,8)$; if the proportion reported was larger, it would mean fewer women were infected during the epidemic, and hence fewer would be expected APOs (online Technical Appendix, Sensitivity Analysis on Fraction of Cases Reported section).

Finally, Brazil made Zika notifiable in November 2015, which might have increased reporting (13). In addition, the Zika incidence data varied markedly by region, which may be due to true differences in outbreak dynamics or to differences in reporting of cases (Figure 1). Although variability in weekly Zika incidence data would alter the precise relationship between Zika cases and population-level rate of APO, the general shape and duration of enhanced risk estimated in the model remains the same (online Technical Appendix, Sensitivity Analysis on Fraction of Cases Reported section).

\section{Conclusions}

Our results suggest that if fetal injury from Zika virus infection can occur across a range of gestational ages, APOs after a Zika outbreak could occur for a long time after the outbreak subsided. This duration is longer than if the risk is assumed to be in the first trimester only $(2,14)$. Combined with epidemiologic reports of APOs collected in Brazil, which show an increase in microcephaly rate at a time inconsistent with first trimester-only risk, evidence is mounting to recommend extended surveillance for APOs and to include a spectrum of outcomes, not only microcephaly $(10,15)$.

Our results suggest that when Zika outbreaks are identified, surveillance and planning for infection-associated APOs might need to focus on a longer period than previously thought. In addition to the potential for APOs several months after an epidemic, the risk period may begin soon after the outbreak is detected. Further studies are crucial to refine the risk for APO during gestation and to ensure pregnant women can be correctly informed of their risk, so that population-level surveillance can be effectively implemented.

Dr. Eggo is a mathematical modeler working on infectious disease dynamics and control at the London School of Hygiene \& Tropical Medicine. Her research interests include severe outcomes of infection and the health inequities and inequalities that can result.

Dr. Kucharski is a mathematical modeler focused on outbreak disease dynamics at the London School of Hygiene \& Tropical Medicine. His research interests include new emerging infections and immunity from individual infection history.

\section{References}

1. Kleber de Oliveira W, Cortez-Escalante J, De Oliveira WTGH, do Carmo GMI, Henriques CMP, Coelho GE, et al. Increase in reported prevalence of microcephaly in infants born to women living in areas with confirmed Zika virus transmission during the first trimester of pregnancy-Brazil, 2015. MMWR Morb Mortal Wkly Rep. 2016;65:242-7. http://dx.doi.org/10.15585/ mmwr.mm6509e2

2. Johansson MA, Mier-y-Teran-Romero L, Reefhuis J, Gilboa SM, Hills SL. Zika and the risk of microcephaly. N Engl J Med. 2016;375:1-4. Erratum in: N Engl J Med. 2016;375:498. http://dx.doi.org/10.1056/NEJMp1605367

3. França GVA, Schuler-Faccini L, Oliveira WK, Henriques CMP, Carmo EH, Pedi VD, et al. Congenital Zika virus syndrome in Brazil: a case series of the first 1501 livebirths with complete investigation. Lancet. 2016;388:891-7. http://dx.doi.org/10.1016/ S0140-6736(16)30902-3

4. Brasil P, Pereira JP Jr, Moreira ME, Ribeiro Nogueira RM, Damasceno L, Wakimoto M, et al. Zika virus infection in pregnant women in Rio de Janeiro. N Engl J Med. 2016;375:2321-34. http://dx.doi.org/10.1056/NEJMoa1602412

5. Cauchemez S, Besnard M, Bompard P, Dub T, Guillemette-Artur P, Eyrolle-Guignot D, et al. Association between Zika virus and microcephaly in French Polynesia, 
2013-15: a retrospective study. Lancet. 2016;387:2125-32. http://dx.doi.org/10.1016/S0140-6736(16)00651-6

6. Kucharski AJ, Funk S, Eggo RM, Mallet H-P, Edmunds WJ, Nilles EJ. Transmission dynamics of Zika virus in island populations: a modelling analysis of the 2013-14 French Polynesia outbreak. PLoS Negl Trop Dis. 2016;10:e004726. http://dx.doi.org/10.1371/journal.pntd.0004726

7. Martinez ME. Preventing Zika virus infection during pregnancy using a seasonal window of opportunity for conception. PLoS Biol. 2016;14:e1002520. http://dx.doi.org/10.1371/journal.pbio.1002520

8. Duffy MR, Chen T-H, Hancock WT, Powers AM, Kool JL, Lanciotti RS, et al. Zika virus outbreak on Yap Island, Federated States of Micronesia. N Engl J Med. 2009;360:2536-43. http://dx.doi.org/10.1056/NEJMoa0805715

9. Honein MA, Dawson AL, Petersen EE, Jones AM, Lee EH, Yazdy MM, et al.; US Zika Pregnancy Registry Collaboration. Birth defects among fetuses and infants of US women with evidence of possible Zika virus infection during pregnancy. JAMA. 2017;317:59-68. http://dx.doi.org/10.1001/jama.2016.19006

10. van der Linden V, Pessoa A, Dobyns W, Barkovich AJ, Júnior HV, Filho ELR, et al. Description of 13 infants born during October 2015-January 2016 with congenital Zika virus infection without microcephaly at birth — Brazil. MMWR Morb Mortal Wkly Rep. 2016;65:1343-8. http://dx.doi.org/10.15585/mmwr.mm6547e2

11. Pacheco O, Beltrán M, Nelson CA, Valencia D, Tolosa N, Farr SL, et al. Zika virus disease in Colombia-preliminary report. N Engl J Med. 2016;NEJMoa1604037. Epub ahead of print. http://dx.doi.org/10.1056/NEJMoa1604037

12. de Oliveira WK, Carmo EH, Henriques CM, Coelho G, Vazquez E, Cortez-Escalante J, et al. Zika virus infection and associated neurologic disorders in Brazil. N Engl J Med. 2017;376:1591-3. http://dx.doi.org/10.1056/NEJMc1608612

13. Brito CA, Brito CC, Oliveira AC, Rocha M, Atanásio C, Asfora C, et al. Zika in Pernambuco: rewriting the first outbreak. Rev Soc Bras Med Trop. 2016;49:553-8. http://dx.doi.org/10.1590/0037-8682-0245-2016

14. Reefhuis J, Gilboa SM, Johansson MA, Valencia D, Simeone RM, Hills SL, et al. Projecting month of birth for at-risk infants after Zika virus disease outbreaks. Emerg Infect Dis. 2016;22:828-32. http://dx.doi.org/10.3201/eid2205.160290

15. Aragao MFVV, Holanda AC, Brainer-Lima AM, Petribu NCL, Castillo M, van der Linden V, et al. Nonmicrocephalic infants with congenital Zika syndrome suspected only after neuroimaging evaluation compared with those with microcephaly at birth and postnatally: how large is the Zika virus "iceberg"? AJNR Am J Neuroradiol. 2017;38:1427-34. http://dx.doi.org/ 10.3174/ajnr.A5216

Address for correspondence: Rosalind M. Eggo, London School of Hygiene \& Tropical Medicine, Keppel Street, London WC1E 7HT, UK; email: r.eggo@1shtm.ac.uk

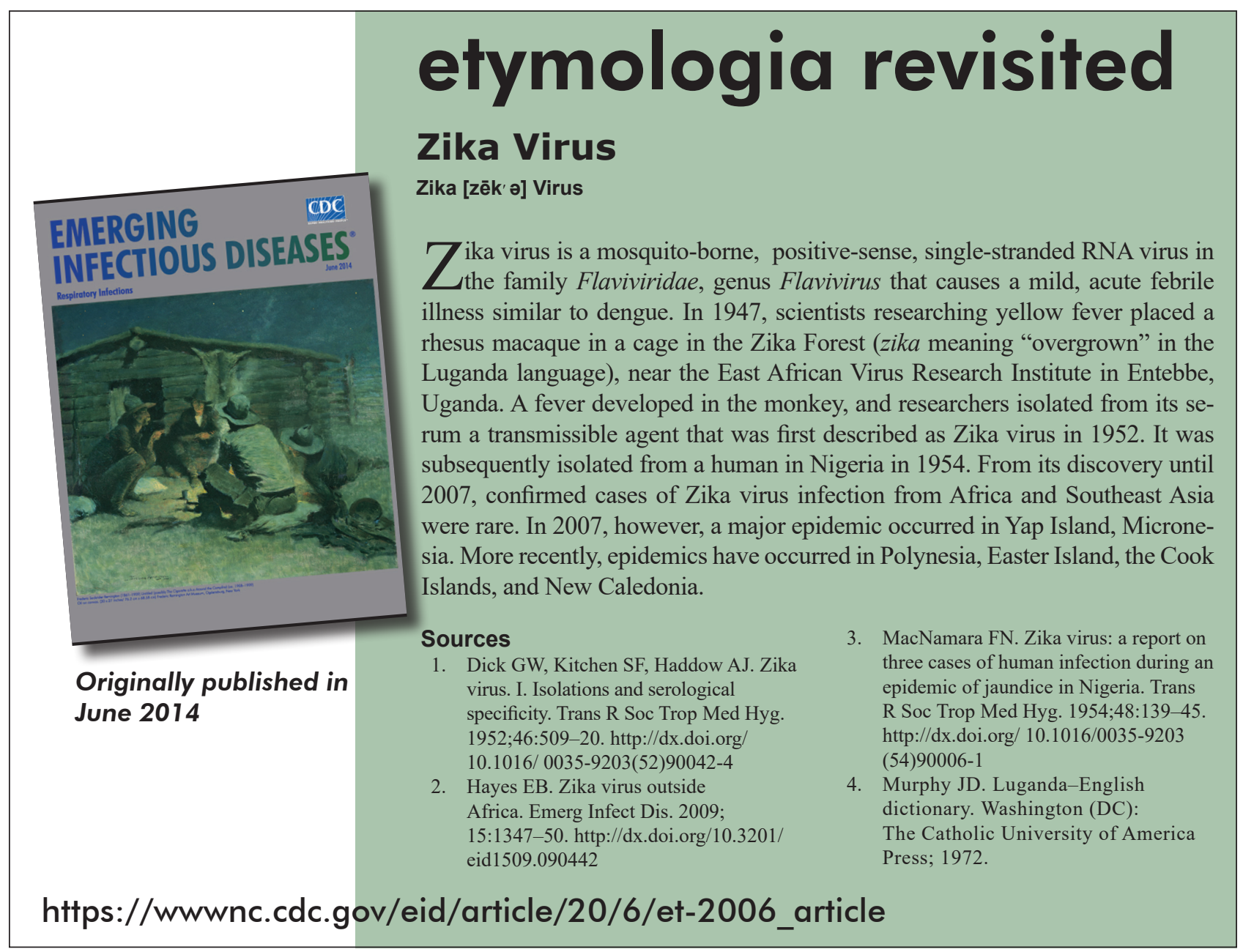

